\title{
Uma Contribuição ao Estabelecimento de Requisitos para Aplicações de Aprendizagem Baseada em TV-Digital
}

\author{
Norben Paulus de Oliveira Costa ${ }^{1}$, Alexandre Freitas Duarte ${ }^{2}$, Nemésio Freitas \\ Duarte Filho $^{3}$, Ellen Francine Barbosa ${ }^{3}$ \\ ${ }^{1}$ União das Instituições Educacionais do Estado de São Paulo (UNIESP) \\ Caixa Postal 14.010-060 - Ribeirão Preto - SP - Brasil \\ ${ }^{2}$ Faculdade de Filosofia Ciências e Letras (FFCLRP/USP) - Universidade de São Paulo \\ Caixa Postal 14.015-000 - Ribeirão Preto - SP - Brasil \\ ${ }^{3}$ Instituto de Ciências Matemáticas e de Computação (ICMC/USP) - Universidade de São Paulo \\ Caixa Postal 13.566-590 - São Carlos - SP - Brasil \\ shadow_suporte@hotmail.com, alexfd7@gmail.com, \{nemesio,francine\}@icmc.usp.br
}

\begin{abstract}
In recent years, the t-learning achieved great importance as a potential means of learning and training for apprentices, ensuring flexibility and convenience to teaching. This paper discusses the proposal of a preliminary set of characteristics and requirements for t-learning applications. A systematic review was conducted in order to understand how the scientific community investigates and describes these characteristics and requirements in the field of education.
\end{abstract}

Resumo. Nos últimos anos, o t-learning atingiu uma grande importância como potencial meio de aprendizagem e treinamento para os aprendizes, garantindo flexibilidade e comodidade ao ensino. O presente trabalho discute a proposta de um conjunto preliminar de características e requisitos específicos para aplicações de t-learning. Uma revisão sistemática foi realizada com o intuito de compreender como a comunidade científica descreve $e$ investiga tais características e requisitos neste domínio educacional.

\section{Introdução}

Nos últimos anos, temas relacionados ao ensino e aprendizagem têm sido cada vez mais discutidos e estudados pela comunidade científica. Com a evolução da tecnologia, novas formas de aprendizagem estão emergindo, proporcionando maiores facilidades e comodidade aos aprendizes e seus tutores [Olsevicova 2007].

Neste contexto, com o advento da TV Digital, o televisor tornou-se não só um aparelho de radiodifusão convencional, mas um sistema com características flexíveis, sendo relacionadas à capacidade de execução de aplicativos e interatividade, podendo ser aplicada diretamente para o ensino e aprendizagem contemporânea [Junior et al. 2012]. A utilização da TV Digital para fins educacionais proporcionou uma nova modalidade de ensino, denominada t-learning ( $T V$-based interactive learning), oferecendo novas experiências de ensino a distância. A tecnologia da televisão digital 
traz grandes benefícios para as práticas pedagógicas e educacionais [Lytras et al. 2002]: (1) dando apoio à execução de recursos gráficos de alta qualidade; (2) controle e gerenciamento através de um controle remoto; (3) interação elementar, não necessitando aplicar objetos de aprendizagem complexos; (4) motivação e comodidade no ensino; e (5) apresentação e renderização das informações em alta resolução. Apesar dos benefícios ao ensino, por ser um conceito novo e ainda incipiente, o t-learning apresenta algumas limitações em sua construção e utilização.

Como a televisão é um meio de comunicação, ela pode ser estudada através das áreas de teoria da comunicação e da mídia. Em termos de aspectos técnicos, ficam a cargo das engenharias e das ciências da computação [Belda 2009], existindo preocupações relacionadas a taxas de transmissão das informações, segurança, confiabilidade, aspectos de usabilidade, carência de padrões arquiteturais e falta de padronização em relação a requisitos e características específicos para as práticas educacionais.

A definição de requisitos específicos para aplicações de t-learning é uma das etapas cruciais para o sucesso desses sistemas. De fato, além de levar em consideração aspectos técnicos, necessidades intrínsecas relacionadas ao dia a dia dos aprendizes e tutores, sendo elas: educacionais, sócio-culturais e econômicas, também precisam ser consideradas, de modo a permitir maior qualidade e eficiência para o ensino. Dentro desse contexto, objetivo deste trabalho é propor e definir um conjunto preliminar de requisitos específicos ao domínio da aprendizagem baseada em TV Digital, facilitando o desenvolvimento e padronização desses ambientes.

O presente trabalho encontra-se subdividido em cinco seções. A Seção 2 apresenta a fundamentação teórica sobre t-learning. A Seção 3 detalha a metodologia de pesquisa adotada no trabalho, conduzida por meio de uma Revisão Sistemática. A Seção 4 traz a análise e discussão dos resultados obtidos a partir da Revisão Sistemática, descrevendo as características e requisitos específicos para ambientes de t-learning. Por fim, a Revisão Sistemática apresenta a conclusão e trabalhos futuros.

\section{T-Learning}

O t-learning (TV-based interactive learning) consiste na utilização de conteúdos interativos com base na televisão. Os ambientes de t-learning procuram atingir audiências mais vastas em regiões remotas e também podem oferecer conteúdos on-line e apoio à formação de uma rede de educação a distância [Barbosa 2007].

Segundo [Belda 2009], a TV Digital vai além de "imagens limpas" e melhor qualidade de áudio, é uma eventual convergência da televisão, telefonia, internet e computador pessoal em um simples equipamento doméstico, com a expectativa de acesso amplo a todos os tipos de informação e comunicação interativa. A expressão $t$ learning descreve a união entre a TVDI (TV Digital Interativa) e o uso de tecnologia computacional para dar apoio ao treinamento e atividades educacionais [Feng et al. 2012]. A união de elementos como televisão, computadores e das práticas do $e$-learning permitem aos usuários combinar a capacidade multimídia da TV com a interatividade e a personalização oferecidas pelo ambiente computacional, em especial a internet [Lytras et al. 2002]. 
Um ponto a se ressaltar é que na transmissão linear e tradicional da televisão analógica não se permite nenhum retorno ao usuário, impossibilitando-lhe interagir com o conteúdo assistido [Wang, 2012]. A concepção de um sistema de TV totalmente digital, batizado de DTV (Digital Television) iniciou-se nos Estados Unidos em 1987.

No caso da TVDI, aplicações interativas permitem ao usuário interagir com o conteúdo, as interações podem ser classificadas em três níveis [Véras 2011]: (1) o primeiro e mais básico é a interação com a TV para escolher algo na programação, grava-la ou assisti-la sob demanda; (2) em um segundo nível, o telespectador poderia alterar a programação pelo controle remoto, podendo, por exemplo, mudar o final de um filme; e (3) já o terceiro nível representaria uma mudança significativa na forma de se relacionar com os aparelhos televisivos, permitindo acesso a informações adicionais do programa assistido, seja em texto ou vídeo, e a definição de um perfil para o telespectador.

Entretanto, apesar dos benefícios oferecidos e mesmo existindo um exponencial aumento em pesquisas relacionadas ao desenvolvimento de ambientes de t-learning, poucos são os trabalhos que apresentam um quadro comum e comparativo em relação às características e requisitos de desenvolvimento neste novo contexto de ensino [Lytras et al. 2002; Belda 2009]. Além disso, as iniciativas existentes encontram-se dispersas e não são padronizadas [Olsevicova 2007; Mathur et al. 2011; Junior et al. 2012; Ayad 2010].

A identificação e o entendimento de características e requisitos específicos para ambientes de t-learning é uma tarefa complexa [Jiang et al. 2011; Claros and Cobos 2012]. Diferentes fatores estão envolvidos no desenvolvimento desses ambientes, os quais devem levar em consideração, além de aspectos técnicos, atributos educacionais, sociais, econômicos e culturais. Para investigar e sintetizar os aspectos relacionados às características e requisitos para ambientes de t-learning, uma Revisão Sistemática foi planejada e executada. A idéia foi obter evidências fundamentadas sobre o tema em questão, de modo, a compreender como a comunidade científica investiga e descreve tais características e requisitos para este novo domínio educacional.

\section{Revisão Sistemática sobre TV-Based Interactive Learning}

Conceitualmente, Revisão Sistemática é uma metodologia utilizada para realizar revisões bibliográficas de forma organizada, permitindo a sua condução de forma sistemática através de etapas bem definidas, proporcionando maior embasamento e confiabilidade científica [Kitchenham 2004].

Nesta seção são detalhadas as etapas de planejamento e condução de uma revisão sistemática no contexto de ambientes t-learning, os resultados obtidos a partir de sua realização também são sintetizados.

\subsection{Planejamento da Revisão Sistemática}

Considerando o contexto de execução deste trabalho, na etapa de planejamento foi definido o protocolo da revisão, ou seja, foram propostas e levantadas questões ligadas à pesquisa, sendo feita a seleção das fontes de informações e, por fim, a seleção dos estudos. A Tabela 1 sintetiza os objetivos principais e secundários da pesquisa. 
Tabela 1 Objetivos Principais e Secundários

\begin{tabular}{|l|ll|}
\hline \multicolumn{1}{|c|}{ Objetivos Principais } & \multicolumn{1}{|c|}{ Objetivos Secundários } \\
\hline $\begin{array}{l}\text { Identificar as principais características presentes em } \\
\text { ambientes de } \text {-learning. }\end{array}$ & Analisar ambientes de -learning. \\
\hline $\begin{array}{l}\text { Analisar as tendências e padrões relacionados à } \\
\text { ambientes de } \text { t-learning. }\end{array}$ & $\begin{array}{l}\text { Analisar características e requisitos de qualidade } \\
\text { específicos para estes ambientes. }\end{array}$ \\
\hline $\begin{array}{l}\text { Identificar as principais funcionalidades (requisitos } \\
\text { funcionais/não-funcionais) de um ambiente de } \\
t \text {-learning. }\end{array}$ & $\begin{array}{l}\text { Verificar as principais dificuldades e limitações } \\
\text { associadas aos ambientes de t-learning. }\end{array}$ \\
\hline
\end{tabular}

De acordo com Kitchenham [Kitchenham 2004], no contexto do processo de uma Revisão Sistemática, a construção das questões de pesquisa é de extrema importância para estabelecer o foco de interesse do trabalho, pois as respostas em relação às questões ajudam a obter uma conclusão e análise específica sobre o problema em questão. As questões para a pesquisa foram levantadas e exibidas na Tabela 2.

Além disso, para que uma questão de pesquisa possa ser bem formulada ela deve ser composta por quatro itens, identificados como PICO (Population, Intervation, Comparison, Outcome). A partir das questões construídas, as seguintes especificidades foram consideradas: (1) Population, pesquisas relacionadas à construção e desenvolvimento de ambientes de t-learning; (2) Intervation, características e requisitos de desenvolvimento; (3) Comparison, avaliar e analisar diversas características e requisitos presentes em ambientes de TV Digital vinculados ao ensino e aprendizagem; e (4) Outcome, identificação de características necessárias para a construção de um ambiente de t-learning, que seja eficiente ao ensino e aprendizagem, facilitando a sua utilização por parte de tutores e aprendizes.

Tabela 2 - Questões da Pesquisa

Questão 1: Quais as principais características presentes em ambientes de t-learning?

Questão 2: Quais os principais requisitos (funcionais/não-funcionais) necessários para a construção de ambientes de $t$-learning?

Questão 3: Existem técnicas e padrões arquiteturais que facilitam o desenvolvimento destes ambientes?

Após a definição das questões de pesquisa, foi definida a estratégia de busca, para isso foram definidos critérios para a seleção das fontes de busca, palavras chaves e strings de busca. Alguns critérios foram definidos em relação às fontes de busca. As fontes de busca foram escolhidas levando em consideração o impacto e a abrangência na área da computação e educação. A partir dos critérios analisados, foram escolhidas como fontes de pesquisa: IEEE Xplore, ACM Digital Lybrary, Springer Link, Scirus, Scopus e ISI Web Knowledge. Por fim, antes da condução da revisão sistemática também é relevante e necessária a definição de critérios de Inclusão (CI) e Exclusão (CE). A Tabela 3 sintetiza os critérios estabelecidos.

Tabela 3 - Critérios de Inclusão e Exclusão.

\begin{tabular}{|l|l|l|}
\hline \multicolumn{1}{|c|}{ Critérios de Inclusão (CI) } & \multicolumn{1}{|c|}{ Critérios de Exclusão (CE) } \\
\hline $\begin{array}{l}\text { Estudos primários que contemplam aplicações e } \\
\text { análises de ambientes de } t \text {-learning; }\end{array}$ & $\begin{array}{l}\text { Estudos primários que evidenciam características e } \\
\text { requisitos de ambientes de } \text { t-learning sem embasamento } \\
\text { teórico ou prático; }\end{array}$ \\
\hline $\begin{array}{l}\text { Estudos primários em relação a características e } \\
\text { funcionalidades específicas para ambientes de t- } \\
\text { learning; }\end{array}$ & $\begin{array}{l}\text { Estudos primários que analisam somente requisitos em } \\
\text { relação a ambientes de } t \text {-learning; }\end{array}$ \\
\hline $\begin{array}{l}\text { Estudos primários que estejam ligados a questões e } \\
\text { dificuldades em relação à construção e } \\
\text { desenvolvimento destes ambientes. }\end{array}$ & $\begin{array}{l}\text { Estudos primários que estejam em idiomas diferentes do } \\
\text { inglês e português, dificultando a coleta e a } \\
\text { identificação de informações. }\end{array}$ \\
\hline
\end{tabular}




\subsection{Condução da Revisão Sistemática}

$\mathrm{Na}$ etapa subseqüente, o processo de construção da string de busca exige cuidado, pois sua definição está ligada diretamente aos objetivos, escopo e resultados da pesquisa. Antes da definição da string de busca, testes foram efetuados nas próprias bases de dados, utilizando alguns sinônimos definidos nas palavras-chaves, verificando a forma e a quantidade de resultados encontrados. Tendo como base os testes efetuados, a string genérica utilizada nas fontes de pesquisa foi definida da seguinte maneira:

\section{t-learning OR edutainment AND ("requirements" OR "characteristics")}

Tendo como base a string genérica, foram construídas strings específicas para cada máquina de busca. As buscas foram realizadas a partir das palavras-chaves, sendo inseridas no título e no abstract dos trabalhos em relação às bases de dados definidas na seção anterior. Verificou-se, ainda, que algumas bases não disponibilizavam opções de busca em relação ao título e abstract. Devido a essa limitação, optou-se por realizar uma pesquisa mais coerente utilizando funções de busca através de keywords e topic.

A partir das buscas realizadas, 329 documentos foram retornados. Após a análise, foram selecionados 132 trabalhos, os quais estavam integralmente disponíveis para leitura (Tabela 4).

Tabela 4 - Resultado Parcial.

\begin{tabular}{|c|c|}
\hline Base de Dados & Resultado \\
\hline IEEE Xplore & 28 \\
\hline ACM Digital Lybrary & 18 \\
\hline Springer Link & 23 \\
\hline Scopus & 34 \\
\hline Scirus & 3 \\
\hline ISI web knowledge & 26 \\
\hline Resultado Final & $\mathbf{1 3 2}$ \\
\hline
\end{tabular}

Após a leitura dos resumos dos 132 estudos primários e a aplicação dos critérios de inclusão e exclusão, o número de trabalhos foi reduzido para 22. Excluindo-se os trabalhos repetidos entre as bases, 17 artigos foram selecionados ao final do processo. A relação das bases e artigos selecionados é sintetizada na Tabela 5.

Tabela 5 - Trabalhos Selecionados.

\begin{tabular}{|c|c|c|}
\hline Referência & Fonte & Título do Trabalho \\
\hline $\begin{array}{l}\text { [Patron-Perez et al. } \\
\text { 2012] }\end{array}$ & $\begin{array}{l}\text { ACM Digital } \\
\text { Lybrary }\end{array}$ & $\begin{array}{l}\text { Structured Learning of Human Interactions in TV } \\
\text { Shows. }\end{array}$ \\
\hline [Jokipelto 2006] & $\begin{array}{l}\text { ACM Digital } \\
\text { Lybrary }\end{array}$ & $\begin{array}{l}\text { Modeling and Content Production of Distance } \\
\text { Learning Concept for Interactive Digital Television. }\end{array}$ \\
\hline [Belda 2009] & IEEE Xplore & $\begin{array}{l}\text { Um modelo estrutural de conteúdos educativos para } \\
\text { televisão digital interativa. }\end{array}$ \\
\hline [Olsevicova 2007] & IEEE Xplore & $\begin{array}{l}\text { t-learning Approach: Enhancing Video with Active } \\
\text { Elements. }\end{array}$ \\
\hline [Ayad 2010] & IEEE Xplore & $\begin{array}{l}\text { The role of edutainment in e-learning: An empirical } \\
\text { study. }\end{array}$ \\
\hline [Lee 2012] & IEEE Xplore & $\begin{array}{l}\text { Dreamware: edutainment system for children with } \\
\text { developmental disability. }\end{array}$ \\
\hline [Douadi et al. 2012] & IEEE Xplore & Smart edutainment game for algorithmic thinking. \\
\hline [Jiang 2011] & IEEE Xplore & $\begin{array}{l}\text { Interactive System for t-learning: Introduction of iTV } \\
\text { Edutainment Mode. }\end{array}$ \\
\hline [Junior et al. 2012] & IEEE Xplore & $\begin{array}{l}\text { The Interactive Digital TV based on Distance } \\
\text { Education: Integrated Collaboration Environments. }\end{array}$ \\
\hline [Narvaez et al. 2012] & Scirus & $\begin{array}{l}\text { Usability guidelines related to ease of learning and } \\
\text { ease of use in the design of interactive digital }\end{array}$ \\
\hline
\end{tabular}




\begin{tabular}{|l|l|l|}
\hline $\begin{array}{l}\text { [Claros and Cobos } \\
\text { 2012] }\end{array}$ & Scirus & $\begin{array}{l}\text { television applications, considering user profiles. } \\
\text { An approach for t-learning content generation based } \\
\text { on a social media environment. }\end{array}$ \\
\hline $\begin{array}{l}\text { [Feng et al. 2012] } \\
\text { [Baum and Soares } \\
\text { Construction and Application of Learning Petri Net. }\end{array}$ & Scirus & Scirus \\
\hline [Lopez et al. 2012] & $\begin{array}{l}\text { ISI Web } \\
\text { Knowledge }\end{array}$ & $\begin{array}{l}\text { Cycle of PDCA t-learning Model and its Application } \\
\text { on Interactive Digital TV. }\end{array}$ \\
\hline [Wang 2012] & Scopus & $\begin{array}{l}\text { Towards Web-Based Adaptive Learning Assistant } \\
\text { Approach. }\end{array}$ \\
\hline [Mathur et al. 2011] & Scopus & $\begin{array}{l}\text { Interactive TV: A step ahead for imparting education } \\
\text { in India. }\end{array}$ \\
\hline [Shetty 2012] & Springer Link & Interactive t-learning system. \\
\hline
\end{tabular}

Os 17 estudos foram lidos e analisados em conformidade com os objetivos e as questões definidas inicialmente. Para cada trabalho analisado os autores identificaram características e requisitos relevantes aos ambientes t-learning, os quais são descritos e detalhados na próxima seção.

\section{Conjunto Preliminar de Requisitos}

A definição de requisitos em relação a um domínio específico de software ajuda não somente na modelagem, padronização e manutenção, mas também na garantia da qualidade e na eficiência do sistema. Além disso, no contexto do t-learning é necessário projetar critérios específicos, permitindo que os atributos da TV Digital, possam atender corretamente aos requisitos educacionais [Mathur et al. 2011; Narvaez et al. 2012].

Os resultados iniciais possibilitaram a proposta de um conjunto preliminar de requisitos específicos para aplicações em t-learning estruturados/clusterizados em quatro dimensões: (1) técnico; (2) educacional; (3) sócio-cultural; e (4) econômico. Estes grupos estão vinculados aos requisitos e características identificados nos trabalhos da revisão sistemática. A Clusterização dos documentos (Figura 1), juntamente com a identificação de sua correlação foi realizada através da Revis Tool (Systematic Review Supported by Visual Analytics) [Felizardo et al. 2012], ferramenta utilizada para apoiar a seleção e avaliação de qualidade de estudos primários.

O grupo (1-Técnico) refere-se a artigos que abordam atributos relacionados à funcionalidades, usabilidade, troca de comunicações, segurança e aspectos de confiabilidade e disponibilidade, tendo como palavras similares deste grupo (Security; Usability; Transmission; Confiability). O segundo grupo (2-Educacional) relaciona-se com características e requisitos educacionais, flexibilizando a interação, acessibilidade e motivação por partes de seus usuários, sendo eles tutores e aprendizes, as palavras similares deste grupo são (Context-aware; Feedback; Interaction; Accessibility). O terceiro grupo (3-Sócio Cultural) relaciona-se com elementos que facilitam a aceitação e utilização destes ambientes, as palavras similares deste grupo são (Cultural Tendency; Transparency; Social Interactions; Acceptability); Por fim, no quarto domínio (4Econômico) os trabalhos se relacionam com abordagens relacionadas ao custo $\mathrm{x}$ benefícios destes ambientes, tendo como palavras-chaves (Viability; Service; Contract Guarantees; Cost; Benefits). 


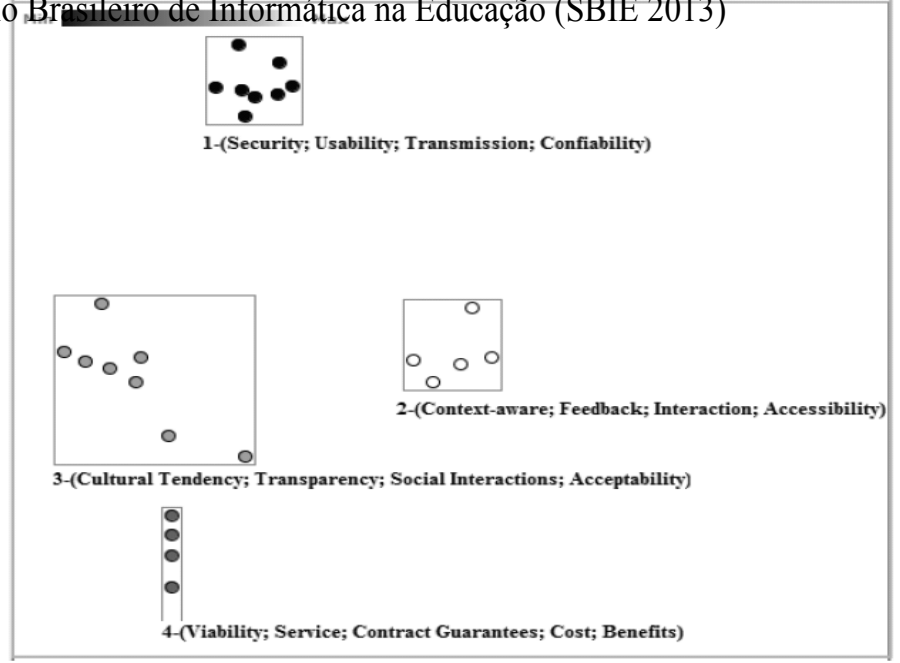

Figura 1: Agrupamento dos documentos da Revisão Sistemática.

As características identificadas na pesquisa foram relacionadas com requisitos apropriados, identificados durante a análise da Revisão Sistemática. Ao final de todo o processo, o "mapa mental" contendo as características e os requisitos relevantes aos ambientes de t-learning pode ser visualizado pela Figura 2. As subseções seguintes identificam os requisitos, definindo-os em relação ao domínio de aprendizagem interativa baseada em TV-Digital.

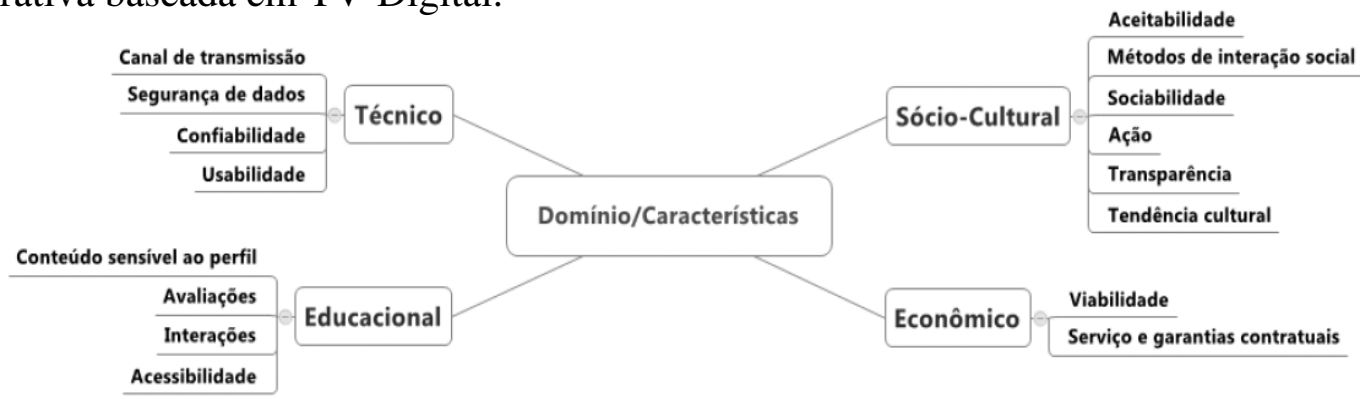

Figura 2: Mapa Mental: Domínio e Características para TV-Based Interactive Learning.

\subsection{Técnico}

Os aspectos tecnológicos representam premissas e restrições quanto à arquitetura tecnológica, padrões, comunicação, segurança, entre outros. A Tabela 6 sintetiza os principais recursos tecnológicos envolvidos nas práticas de $t$-learning.

\begin{tabular}{|l|l|}
\multicolumn{2}{l}{ Tabela 6 - Requisitos técnicos para t-learning. } \\
\hline Requisitos & Definição \\
\hline Canal de transmissão & $\begin{array}{l}\text { Necessidade de meios de comunicação eficientes utilizados para transmitir as } \\
\text { informações para dispositivos fixos ou móveis [Narvaez et al. 2012; Ayad } \\
2010] .\end{array}$ \\
\hline Segurança de dados & $\begin{array}{l}\text { Alta prioridade para aspectos de integridade, autenticação e privacidade dos } \\
\text { dados e informações [Baum and Soares 2012]. }\end{array}$ \\
\hline Confiabilidade & $\begin{array}{l}\text { Testes devem ser realizados com objetivos de prover serviços com alta } \\
\text { performance e qualidade de vídeo e áudio [Belda 2009; Claros and Cobos } \\
2012] .\end{array}$ \\
\hline Usabilidade & $\begin{array}{l}\text { A navegação pelo conteúdo didático deve ser clara e intuitiva. A exibição e } \\
\text { renderização das informações devem ser realizadas de maneira eficiente em } \\
\text { diferentes aparelhos televisuais [Douadi et al. 2012; Feng et al. 2012]. }\end{array}$ \\
\hline
\end{tabular}

\subsection{Educacional}

Os requisitos educacionais têm por objetivo, garantir maior competência e adaptação às práticas educacionais, flexibilizando as atividades e tarefas dos aprendizes. Os aspectos educacionais fundamentais para aplicações em t-learning são mostrados na Tabela 7. 
Tabela 7 - Requisitos educacionais para t-learning.

\begin{tabular}{|l|l|}
\hline Requisitos & Definição \\
\hline Conteúdo sensível ao perfil & $\begin{array}{l}\text { Suporte e personalização baseado no contexto dos usuários mediante ao } \\
\text { ambiente, atividades e outras características [Jiang et al. 2011; Jokipelto 2006]. }\end{array}$ \\
\hline Avaliações & $\begin{array}{l}\text { Pode e deve ser realizada através de diferentes formas, sendo elas: colaborativa, } \\
\text { individual, somativa, entre outras [Junior et al. 2012; Lee 2012]. }\end{array}$ \\
\hline Interações & $\begin{array}{l}\text { Suporte às interações (síncronas e assíncronas) entre os aprendizes, tutores, } \\
\text { objetos e ambientes de aprendizagem [Lopez et al. 2012; Lytras et al. 2002]. }\end{array}$ \\
\hline Acessibilidade & $\begin{array}{l}\text { Deve permitir que os programas educacionais para televisão possam ser } \\
\text { estruturados de forma linear (a forma do curso é determinada pelo tutor) e não } \\
\text { linear (o aprendiz pode navegar da forma que melhor lhe convier) [Mathur et } \\
\text { al. 2011]. }\end{array}$ \\
\hline
\end{tabular}

\subsection{Sócio-Cultural}

Aspectos sócio-culturais garantem adequação aos fatores que dizem respeito a um determinado grupo social e ao nível cultural dos indivíduos que realizam as práticas educacionais. A Tabela 8 mostra os requisitos identificados nesta pesquisa.

Tabela 8- Requisitos sócio-culturais para t-learning.

\begin{tabular}{|l|l|}
\hline Requisitos & Definição \\
\hline Aceitabilidade & $\begin{array}{l}\text { O receptor/aprendiz pode ou não utilizar o t-learning devido a sua condição } \\
\text { social cultural, racial, religiosa, idade, sexo e demais valores morais } \\
\text { [Narvaez et al. 2012; Olsevicova 2007]. }\end{array}$ \\
\hline Métodos de interação social & $\begin{array}{l}\text { Compreende-se um conjunto de expectativas, modos e estilos de linguagem } \\
\text { sociais geradas pelo sistema, sendo elas formais ou informais [Patron-Perez et } \\
\text { al. 2012; Shetty 2012]. }\end{array}$ \\
\hline Sociabilidade & $\begin{array}{l}\text { O sistema deve gerar o desenvolvimento de relacionamentos mútuos entre os } \\
\text { aprendizes e o conteúdo aplicado, promovendo a inclusão de novos } \\
\text { participantes [Véras 2011; Wang 2012]. }\end{array}$ \\
\hline Ação & $\begin{array}{l}\text { Deve elevar o nível de motivação e auto-estima, gerando confiança, } \\
\text { compromisso e eficácia para o ensino e aprendizagem [Patron-Perez et al. } \\
\text { 2012; Shetty 2012]. }\end{array}$ \\
\hline Transparência & $\begin{array}{l}\text { As atividades e dados educacionais não devem ser ocultos aos aprendizes e } \\
\text { tutores, quando solicitados devem estar disponíveis para consultas prévias } \\
\text { [Parker 1999]. }\end{array}$ \\
\hline Tendência cultural & $\begin{array}{l}\text { Característica fundamental para interação dos usuários. As cores e e estilo } \\
\text { geométrico e tridimensional devem traduzir a "moda"e o momento atual para o } \\
\text { espectador, principalmente ligado as "redes sociais" [Olsevicova 2007]. }\end{array}$ \\
\hline
\end{tabular}

\subsection{Econômico}

Neste domínio econômico, existe uma preocupação quanto à viabilidade e garantias frente à utilização de ambientes educacionais, necessitando de um balanceamento entre o custo x benefício da aplicação. A Tabela 9 mostra as principais características econômicas relacionadas às aplicações de t-learning.

Tabela 9- Requisitos econômicos para t-learning.

\begin{tabular}{|l|l|}
\hline Requisitos & Definição \\
\hline Viabilidade & $\begin{array}{l}\text { Traduz a condição principal para a implantação do sistema e sua relação } \\
\text { custo/beneficio para a aprendizagem, tendo como base as fases de compra, } \\
\text { operação, reparo, modernização e riscos que devem ser considerados [Mathur } \\
\text { et al. 2011; Lytras et al. 2002]. }\end{array}$ \\
\hline Serviço e garantias contratuais & $\begin{array}{l}\text { O ambiente educacional deve possibilitar SLA's (Service Level Agreement) } \\
\text { bem definidos. Tais licenças devem estar relacionadas ao número de } \\
\text { participantes, quantidade de conteúdo, disponibilidades, possibilitando } \\
\text { parâmetros importantes para avaliação de qualidade e eficiência [Lee 2012; } \\
\text { Junior, et al. 2012]. }\end{array}$ \\
\hline
\end{tabular}




\section{Conclusão e Trabalhos Futuros}

Este trabalho pretendeu avançar no campo de estudos de TV Digital, no que diz respeito ao tema específico de características e requisitos para aplicações em t-learning, proporcionando um ponto de partida para identificação de tendências, sobreposições, lacunas e desafios nesta área de pesquisa.

Os resultados iniciais da revisão sistemática utilizados em um contexto de aprendizagem interativa baseada em TV Digital proporcionaram um conjunto preliminar de características e requisitos, sendo estruturadas em quatro dimensões fundamentais, podendo ser contemplados durante a fase de elicitação de requisitos juntamente com a sua modelagem, garantindo funcionalidades que irão atender de forma flexível as atividades educacionais vinculadas aos aprendizes e tutores.

O conjunto proposto além de levar em consideração aspectos técnicos, também abrange necessidades intrínsecas relacionadas ao dia a dia dos aprendizes sendo elas: educacionais, sócio-culturais e econômicas. Com isso, espera-se que a aplicação educacional proporcione elementos referentes e necessários à infraestrutura de tecnologia, estratégias didáticas, tendências sócio-culturais e contratuais.

Para trabalhos futuros, os autores pretendem evoluir e melhorar o conjunto de características proposto, proporcionando um conjunto de requisitos de qualidade específicos para aplicações em t-learning. Tal conjunto deve ser estruturado em critérios, requisitos e descrições de qualidade, servindo de guia para as organizações desenvolvedoras, garantindo adequação de qualidade e satisfação às necessidades educacionais e de aprendizagem.

\section{Referências}

Ayad, K. A. A. (2010). The role of edutainment in e-learning: An empirical study. PhD Department of Media Technology, Faculty of Technology, De Montfort University.

Barbosa, A. (2007). O sistema brasileiro de televisão digital: do sonho à realidade. In: TV digital: qualidade e interatividade. Brasília, IEL/NC.

Baum, G., Soares, L. F. G. (2012). Ginga middleware and digital tv in latin america. IT Professional, vol.14, no.4, pp.59-61. July-Aug.

Belda, F. R. (2009). Um modelo estrutural de conteúdos educativos para televisão digital interativa. São Carlos: USP, 292f. Tese (Doutorado) - Escola de Engenharia de São Carlos, Universidade de São Paulo, São Carlos.

Claros, I., Cobos, R. ( 2012). An approach for t-learning content generation based on a social media environment. EuroITV'12, 4-6, Berlin, Germany (in press). July.

Douadi, B., Tahar, B., Hamid, S. (2012). Smart edutainment game for algorithmic thinking. Procedia-Social and Behavioral Sciences, Volume 31, p. 454-458 - Elsevier.

Felizardo, K. R., Salleh, N., Martins, R. M., Mendes, E., Macdonell, S. G., Maldonado, J. C. (2011). Using visual text mining to support the study selection activity in systematic literature reviews. Proceeding ESEM '11 Proceedings of the 2011 International Symposium on Empirical Software Engineering and Measurement Pages 77-86.

Feng, L., Obayashi, M., Kuremoto, T., Kobayashi, K. (2012). Construction and application of learning petri net. http://dx.doi.org/10.5772/48398 Dec. 
Jiang, W., Zuo, J. W. (2011). Interactive system for t-learning: Introduction of itv edutainment mode. Advanced Materials Research (Volume 215),p. Advanced Materials Research (Volume 215) March.

Jokipelto, P. A. (2006). Modeling and content production of distance learning concept for interactive digital television. Dissertation of the Department of Computer Science and Engineering, University of Technology (Espoo, Finland) December.

Junior, I. H. F., Teixeira, M. M., Silva, B. D., Rodrigues, C. M. O., Dias, F. R., Azevedo, R. R. (2012). The interactive digital tv based on distance education: Integrated collaboration environments. Digital Communication Policies.

Kitchenham, B. (2004). Procedures for performing systematic reviews. Relatório Técnico, Keele University and NICTA.

Lee, J. H., Choi, E., Song, M., Shin, B. S. (2012). Dreamware: edutainment system for children with developmental disability. Multimedia Tools and Applications. April.

Lopez, M., Alberto, G., Builes, J., Alberto, J. (2012). Cycle of pdca t-learing model and its application on interactive digital tv. DYNA, vol. 79, núm. 173, pp. 61-70. Universidad Nacional de Colombia Medellín, Colômbia. April.

Lytras, M., Lougos, C., Chizos, P., Pouloudi, A. (2002). Interactive television and elearning convergence: Examining the potential of t-learning. European Conference on e- Learning e Athens:Athens University of Economics and Business.

Mathur, R., Mathur, K., Loonker, S. (2011). Interactive tv: A step ahead for imparting education in india. International Journal of Computer Science \& Engineering Technology (IJCSET). Vol. 2 No. 6 - June.

Narvaez, R., Hurtado, D. J., Solano, A. F., Collazos, C. A., Arciniegas, J. L. (2012). Usability guidelines related to ease of learning and ease of use in the design of interactive digital television applications, considering user profiles. INTERACCION 12 Proceedings of the 13th International Conference on Interacción Persona-Ordenador Article No. 22, 2012.

Olsevicova, K. (2007). T-learning approach: Enhancing video with active elements. CEUR Workshop Proceedings. Crete, Greece, pp 1-6.

Parker, R. (1999): The economics of digital tv future. In D. Gerbarg, The economics, technology and content of digital TV, USA: Kluwer Academic Publishers 1999.

Patron-perez, A., Marszalek, M., Reid, I., Zisserman, A. (2012). Structured learning of human interactions in tv shows. Pattern Analysis and Machine Intelligence, IEEE Transactions on , vol.34, no.12, pp.2441-2453 Dec, 2012.

Shetty, C. K. G. (2012). Interactive t-learning system. Indian J. Edu. Inf. Manage., Vol. 1, No. ISSN 2277 - 53742.

Véras, D., Bittencourt, I., Costa, E. (2011). Construindo aplicações de t-learning integradas com uma plataforma web educacional semântica. In Revista Brasileira de Informática na Educação (RIBIE), v. 19, n. 01.

Wang, H. (2012). Towards web-based adaptive learning assistant approach. Advanced Materials Research, Vol 476-478, p. 2125-2128, 2012. 